\title{
PALOMAR DE PINTADO (VILLAFRANCA DE LOS CABALLEROS, TOLEDO): UN PROYECTO DE FORMACIÓN ACADÉMICA, INVESTIGACIÓN Y REVALORIZACIÓN DE UN YACIMIENTO ARQUEOLÓGICO
}

\author{
THE PALOMAR DE PINTADO PROJECT: ARCHAEOLOGICAL HERITAGE MANAGEMENT \\ IN THE TOLEDO PROVINCE
}

\author{
JESÚS CARROBLES SANTOS $(*)$ \\ JUAN PEREIRA SIESO $(* *)$ \\ ARTURO RUIZ TABOADA (*)
}

\begin{abstract}
RESUMEN
En este trabajo se describe el resultado de la aplicación de un convenio para la excavación de una necrópolis de la Edad del Hierro, entre la Diputación de Toledo, elAyuntamiento de Villafranca de los Caballeros y la Universidad de Castilla La Mancha. La coordinación de estas tres instituciones y su inclusión en un proyecto de desarrollo de centros de interpretación de sitios arqueológicos ha permitido configurar con éxito un nuevo modelo de gestión para una excavación arqueológica.
\end{abstract}

\begin{abstract}
In this paper we present some aspects of a new collaborative research project, focused on the excavation of an Iron Age cemetery, initiated by the Diputación Provincial of Toledo, the local government at Villafranca de los Caballeros and the University of Castilla la Mancha at Toledo. The project provides a common collaborative research framework and involves a new model in the archaeological heritage management.
\end{abstract}

Palabras clave: Gestión de Patrimonio Arqueológico. Necrópolis de incineración. Edad del Hierro. La Mancha.

Key words: Archaeological heritage management. Cremation cemetery. Iron Age. La Mancha.

(*) Servicio de Arqueología. Diputación Provincial de Toledo. Plaza de Abdón de Paz. 45001 Toledo. Correo electrónico: taboada@arrakis.es.

(**) Área de Prehistoria. Facultad de Humanidades de Toledo. Universidad de Castilla-La Mancha (U.C.L.M.). Plaza de Padilla, 4.45071 Toledo. Correo electrónico: jpereira@humto.uclm.es El artículo fue remitido en su versión final el 16-X-2000.

\begin{abstract}
ANTECEDENTES
A comienzos de los años 80 tuvo lugar el descubrimiento casual de una necrópolis ibérica en el término municipal deVillafranca de los Caballeros, como consecuencia de la construcción de un pozo para explotar una pequeña huerta, en el lugar denominado Palomar de Pintado. El hallazgo inicial puso al descubierto principalmente vasos cerámicos de los ajuares funerarios en buen estado de conservación cuya venta a diversos coleccionistas e, incluso, museos locales, propició el inicio de una serie de actuaciones clandestinas, que no pasaron desapercibidas en la localidad y permitieron que se tuviera conocimiento del hallazgo arqueológico.

Ante esta situación y tras una serie de gestiones del alcalde de la localidad encaminadas a la paralización de las actividades clandestinas, justificadas por la ignorancia de la ley, que habían provocado la destrucción parcial de un sector del yacimiento, y tras la obtención del permiso de acceso al terreno para efectuar una evaluación inicial, se pudo comprobar a partir de los restos in situ y a través de los pocos materiales que pudimos conocer el interés del yacimiento en cuestión (Fig. 1). Como conclusión de estas acciones se consideró necesario solicitar un primer permiso de excavación para comprobar la extensión, el estado de conservación y los tipos de estructuras funerarias del yacimiento. Esta actuación se concibió siguiendo el modelo de las actuaciones de urgencia que era uno de los modelos más utilizados en aquellos años para iniciar la intervención en cualquier yacimiento y convertir lo mera-
\end{abstract}




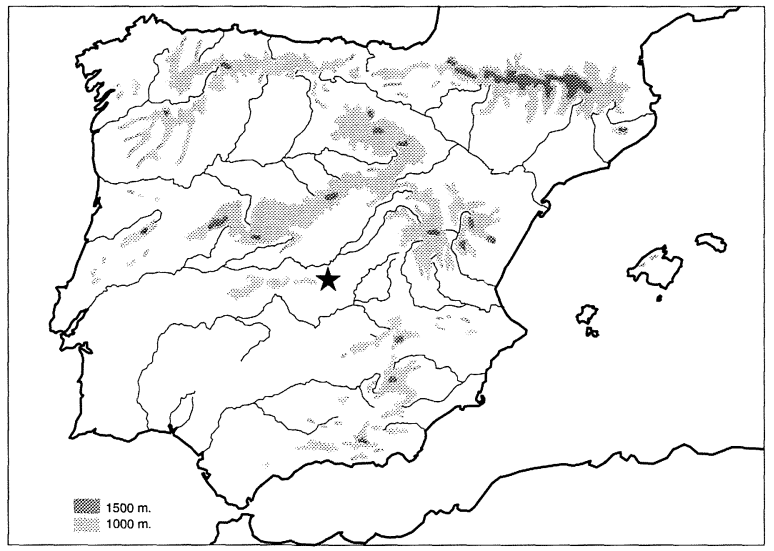

Fig. 1. Localización de la necrópolis del Palomar de Pintado, Villafranca de los Caballeros, Toledo.

mente coyuntural en el inicio de un proyecto a largo plazo, con la única particularidad en nuestro caso de que fue elAyuntamiento de la localidad el que en el año 1986 sufragó, íntegramente, la primera campaña de excavaciones arqueológicas que se llevaron a cabo bajo la dirección de Gonzalo Ruiz Zapatero y Jesús Carrobles Santos (1986).

Estos primeros trabajos pusieron de manifiesto el interés del yacimiento, basado en la excepcional conservación de los conjuntos funerarios y de las estructuras que los contenían, así como en la documentación de nuevos tipos de estructuras funerarias en una zona de la que existían pocos datos sobre lo que ocurrió en la misma durante la Segunda Edad del Hierro.

Los resultados obtenidos en esta primera campaña de excavaciones posibilitaron que la necrópolis de Palomar de Pintado pasara a incluirse dentro del grupo de yacimientos objetos de investigaciones arqueológicas programadas, que comenzaron a ejecutarse en el año 1988 bajo la dirección de Jesús Carrobles Santos, con la colaboración del Ayuntamiento de la localidad, la Junta de Comunidades de Castilla La Mancha y la Diputación Provincial de Toledo.

El mismo esquema de colaboración se repitió en el año 1990, fecha en la que se produjo la adquisición de una parte importante del yacimiento por parte de la Excma. Diputación Provincial de Toledo, con la finalidad de preservar un yacimiento en el que destacaba la excepcional conservación de las estructuras funerarias y sus respectivos ajuares funerarios, puesta de manifiesto a lo largo de los diferentes trabajos efectuados hasta la fecha. Cabe destacar dentro de la preocupación que siempre mantuvimos desde el inicio por divulgar nuestro trabajo la organización de un curso sobre arqueología de la Mancha toledana en el Centro de Profesores de Villacañas coincidiendo con los trabajos de excavación. Gracias a esta iniciativa, los profesores de enseñanza media pertenecientes a los centros implantados en la comarca que lo desearon pudieron participar en la excavación y el resto, en la medidad de su disponibilidad de tiempo pudo programar una serie de visitas a las excavaciones con sus alumnos.

Esta campaña fue la última realizada de acuerdo con un modelo de arqueología tradicional basado en la formación de un pequeño equipo de investigación con el soporte económico de una serie de pequeñas subvenciones oficiales. Estas permitían una intervención anual en el yacimiento, en ocasiones en condiciones precarias, pero sin la posibilidad de llegar a plantear a medio o largo plazo un proyecto de investigación y divulgación que pasara del artículo ocasional o la publicación de materiales o enterramientos que destacaran por su singularidad en el panorama de la Segunda Edad del Hierro de la Meseta Sur (Carrobles y Ruiz Zapatero, 1990; Blasco y Barrio, 1992; Carrobles, 1995).

Ante este estado de cosas, ampliable prácticamente a la totalidad de los yacimientos que se encontraban en proceso de investigación en la provincia de Toledo en los mismos años, estimamos oportuno la paralización de los trabajos hasta no conseguir sacar adelante todo lo obtenido en las anteriores campañas $y$, sobre todo hasta que no se aclarara la función social de nuestro trabajo es decir, que se pudiera llegar a redactar un proyecto en el que la investigación científica fuera acompañada de otra serie de medidas dirigidas a propiciar la conservación de los bienes que integran el yacimiento y a su disfrute en el medio en el que se encontraban. En nuestro criterio una investigación cuyos resultados sólo eran accesibles al restringido circuito del mundo académico e investigador perdería una parte importante de su impacto y justificación sociales, si no éramos capaces de difundirla y hacerla accesible entre la gente que habitaba las localidades cercanas al yacimiento. Este colectivo, de manera creciente, manifestaba curiosidad e interés por visitar y conocer el desarrollo de los trabajos y los resultados que se estaban obteniendo ante las noticias que iban apareciendo en los diferentes medios de comunicación que cubrían la zona. Esta situación se mantuvo hasta el año 1997 en que se pudo configurar un nuevo modelo de ges- 
tión que permitiera la reapertura de las excavaciones en el yacimiento de Palomar de Pintado.

El segundo factor que contribuyó al replanteamiento de este modelo de gestión fue la evolución de los programas de investigación arqueológica desarrollados en el marco universitario del campus de Toledo.

La institucion universitaria que desde principios de los 80 avalaba las campañas de excavaciones arqueológicas en la provincia de Toledo era el Colegio Universitario adscrito inicialmente a la Universidad Complutense que posteriormente fue incorporado a la recien constituida Universidad de Castilla La Mancha. Con la creación de la titulación de Licenciado en Humanidades pasó a denominarse Centro Superior de Humanidades, para convertirse posteriormente en la Facultad de Humanidades del Campus de Toledo. Los proyectos de investigación arqueológica que se desarrollaron durante este proceso presentaban una serie de características que afectaban de manera notable al desarrollo de los mismos. En primer lugar, salvo para las intervenciones coyunturales o de urgencia, el desequilibrio y falta de continuidad en las subvenciones públicas no permitía el desarrollo de programas de investigación a medio y largo plazo. Sólo en el caso del proyecto piloto del Inventario de yacimientos arqueológicos de la provincia de Toledo, encargado en 1983 por la Subdirección General de Arqueología del Ministerio de Cultura, se pudo contar con una subvención importante, que se vió ampliada con la firma de un convenio de colaboración en el proyecto por parte de la Excma. Diputación Provincial de Toledo.

Por otro lado hasta la conversión en Centro Superior de Humanidades, únicamente se impartían en el Colegio Universitario de Toledo las asignaturas del primer Ciclo de la Licenciatura de Historia, por lo que los alumnos debían cursar el Segundo Ciclo fuera del Campus de Toledo. Esta situación se traducía en que alumnos que eran iniciados en el trabajo de campo arqueológico en el marco de distintos proyectos del Colegio Universitario de Toledo, cuando cursaban el segundo Ciclo en otra Universidad se integraban en los proyectos de los Departamentos en que se licenciaban e iniciaban los estudios del Tercer Ciclo.

Esta situación, inevitable por otro lado, impedía que un alumno captado en los primeros años de su carrera, pudiera integrarse en el Colegio Universitario de Toledo en un proyecto a largo plazo del que pudiera aprovechar no sólo la formación teórico- práctica sino también asumir como investigador fases o aspectos del mismo. De otro lado la experiencia nos muestra que las distintas promociones que se incorporan a los estudios universitarios cada curso, presentan notables diferencias en cuanto a su motivación, intereses, preparación, capacidad de trabajo, etc. Estos dos factores fueron determinantes durante años de las dificultades objetivas para crear equipos de investigación más o menos estables.

Las carencias de infraestructura eran también un handicap importante, ya que durante años no se pudo contar con un local que se pudiera utilizar como Laboratorio de Arqueología. En esta situación el simple hecho de almacenar los materiales de una campaña se convertía en un problema, cuanto más las distintas fases de limpieza restauración, documentación y estudio de dichos materiales. Parte de estos problemas se solucionaron temporalmente gracias a la colaboración del Centro de Estudios Internacionales de la Fundación Ortega y Gasset en Toledo que nos permitió durante largas temporadas utilizar parte de sus instalaciones para estos fines.

\section{EL CONVENIO DE COLABORACIÓN}

Retomando el hilo de los antecedentes del cambio de modelo en la gestión del yacimiento de $\mathrm{Pa}$ lomar de Pintado, cabe proseguir señalando que entre el año 1996 y 1997 una serie de contactos y conversaciones entre el responsable del Servicio de Arqueología de la Diputación y el Profesor encargado de las asignaturas de Prehistoria y Arqueología de la Facultad de Humanidades, nos llevó a la constatación de la existencia de una comunidad de intereses.

Por un lado, cada vez era más frecuente que alumnos de la Facultad de Humanidades del Campus de Toledo se presentaran en el Servicio de Arqueología de la Diputación para solicitar su inclusión en cualquier tipo de actividad, ante la falta casi absoluta de prácticas de arqueología de campo. Esta situación era la causante de que desde la aparición de la titulación de Humanidades ningún estudiante de la misma hubiera accedido al mercado laboral como arqueólogo, a pesar del aumento de la demanda de este tipo de profesionales producido en estos años con el crecimiento de la denominada arqueología de gestión y de que, al inicio de los estudios, muchos de los estudiantes solicitaban 
información de cómo aumentar su formación en esta especialidad.

La Facultad de Humanidades contaba ya con un Laboratorio de Arqueología y se habían iniciado una serie de inciativas destinadas a la formación de grupos de alumnos en los trabajos básicos de laboratorio, pero faltaba la posibilidad de acceder a un yacimiento arqueológico con unas ciertas garantías de continuidad en el medio y el largo plazo.

El Servicio de Arqueología de la Diputación de Toledo contaba como se ha referido con un yacimiento arqueológico en propiedad, con un nivel de conservación de estructuras y elementos de cultura material excelente, amén de los materiales procedentes de las primeras campañas, que habían sido estudiados y publicados muy someramente. Sin embargo, su organigrama y la ampliación de sus competencias a los diferentes aspectos de la política cultural de la Diputación Provincial no permitían avanzar en un yacimiento, que había sido incluído en un interesante proyecto de la propia Diputación consistente en un red de pequeños museos que pretendían la revalorización de una serie de bienes culturales de distinta entidad, yacimientos arqueológicos, colecciones particulares, legados, etc ( $\mathrm{Pa}-$ lomero y Carrobles, 1999).

Sobre este estado de cosas empezamos a mantener contactos entre todas las administraciones que habían tenido algo que ver con el yacimiento, con el fin de plantear un nuevo proyecto que hiciera posible la gestión de las posibilidades del yacimiento arqueológico dentro de un marco claramente diferente en el que se podían armonizar aspectos académicos de formación e investigación, aspectos relacionados con una nueva estructura museística, y aspectos relacionados con la difusión y rentabilidad social de un bien cultural en el ámbito de su territorio más inmediato

Este nuevo modelo de gestión se concretó en la firma de un convenio de colaboración entre la Diputación Provincial de Toledo, el Ayuntamiento de Villafranca de los Caballeros y la Universidad de Castilla la Mancha, por el que se reiniciaban las actuaciones en el yacimiento como campo de prácticas para los alumnos de la Facultad de Humanidades del campus toledano, ahora bajo la dirección de Jesús Carrobles Santos, Juan Pereira Sieso y Arturo Ruiz Taboada. Su ejecución sería posible gracias a la sinergia de las aportaciones de las tres instituciones implicadas: Diputación Provincial, Universidad y Ayuntamiento de Villafranca de los Caballeros, que integraban en un mismo proyecto infraestruc- turas, medios materiales y personal responsable de la ejecución del mismo.

La Diputación se responsabiliza de la principal aportación económica en dos de los capítulos de mayor gasto en cualquier excavación como son la manutención de un equipo de 15 personas durante las tres semanas que dura cada campaña según se estipula en el convenio, y los gastos de restauración de los materiales arqueológicos, aportando su Laboratorio de Restauración en el Servicio deArqueología y el personal titulado responsable del mismo. También se hace cargo, previo informe razonado del equipo de dirección del proyecto, de los gastos destinados a cubrir la realización de determinados protocolos analíticos como los análisis de paleodieta.

La Universidad aporta los alumnos de la Facultad de Humanidades de Toledo, procedentes de los dos Ciclos de la Licenciatura. En el tiempo que lleva funcionando el convenio, se ha podido vertebrar un equipo con diferentes niveles de formación y experiencia tanto en los trabajos de campo como en los de laboratorio. Estas labores de documentación se realizan en el Laboratorio de Arqueología de reciente construcción y equipamiento del edificio de la Facultad de Humanidades, que sirve también de almacén y depósito temporal de los materiales arqueológicos así como de archivo de la documentación y bibliografía del yacimiento.

Para afrontar los gastos derivados del trabajo de campo, consolidación y documentación de los materiales así como la consolidación de un equipamiento básico -topografía, fotografía, equipos y programas informáticos y dibujo etc.- desde el área de Prehistoria de la Facultad de Humanidades se gestionan anualmente, avalados por los compromisos asumidos en el convenio, distintos tipos de ayudas a partir de los presupuestos destinados a la investigación universitaria por distintas instituciones o convenios de colaboración entre ellas: Vicerrectorado de Investigación de la UCLM; Patronato Universitario de Toledo y convenio Universidad de Castilla La Mancha y Caja Castilla la Mancha.

ElAyuntamiento de Villafranca de los Caballeros, retomando su antiguo compromiso e interés por el yacimiento, en las fechas consignadas en el convenio pone a total disposición del equipo de excavación el Aula de la Naturaleza para su alojamiento. Este edificio se encuentra en en el area de recreo y turismo del complejo lagunar de Villafranca, en el límite sur de la reserva natural de Las Lagunas (Lám. I). En él se desarrollan también durante la campaña de excavaciones, conferencias, talleres de

T. P., 57, n. $^{\circ} 2,2000$ 

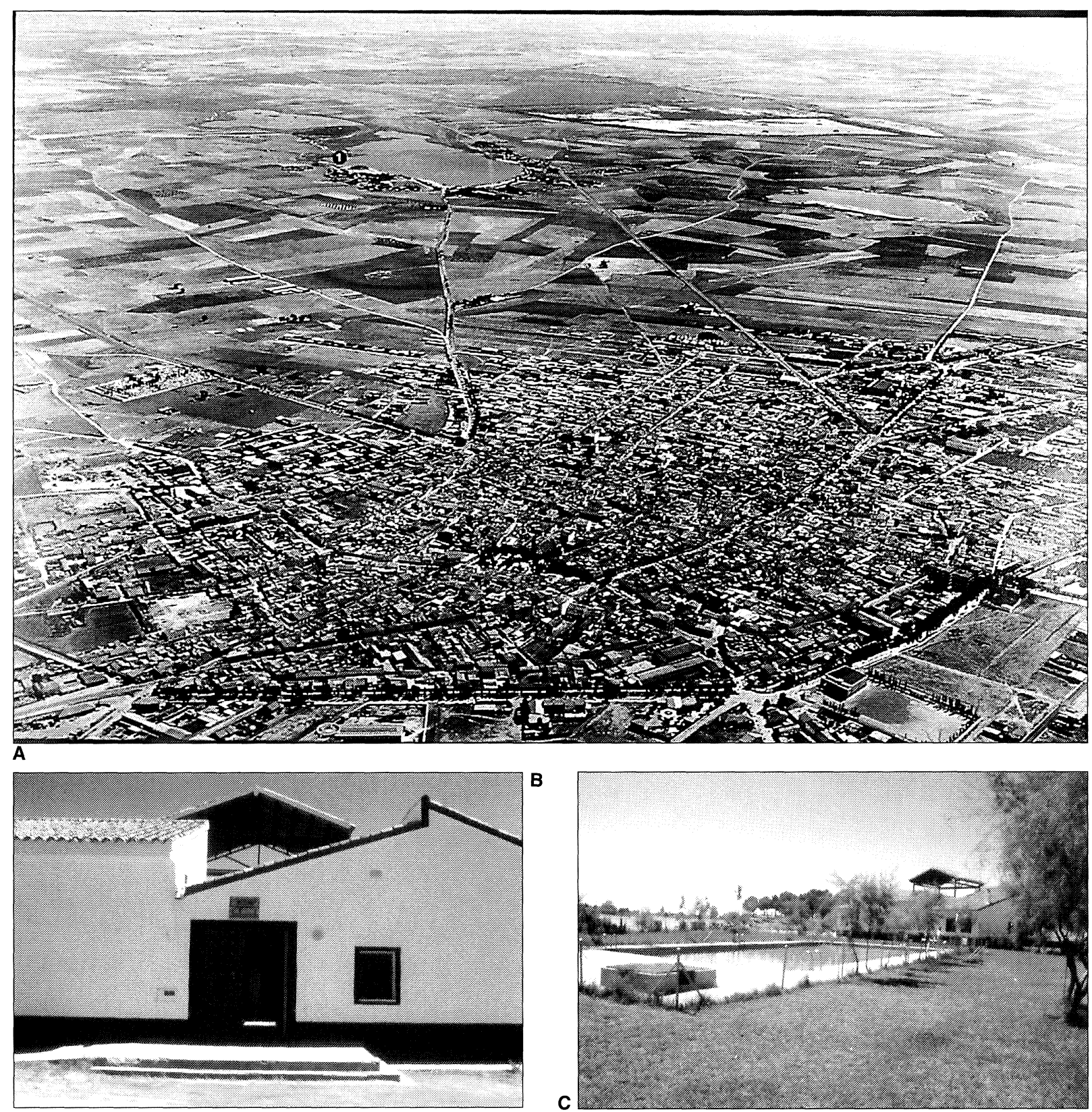

Lám. I.A) Vista aérea de Villafranca de los Caballeros (Toledo) y su complejo lagunar. 1, Aula de la Naturaleza. 2, Casa del Norte. B)Acceso principal al Aula de la Naturaleza de Villafranca de los Caballeros. C)Acceso posterior a sus austeras instalaciones.

limpieza y consolidación de materiales arqueológicos, seminarios sobre prospección arqueológica y funciona también como centro de exposición temporal que más adelante comentaremos.

El Ayuntamiento también se hace cargo de los gastos y necesidades de transporte diario del equipo y de todo lo relacionado con los materiales fungibles necesarios en la excavación, junto con el em- pleo puntual de maquinaria pesada para determinadas labores como el traslado de las terreras y el tapado de los cortes al finalizar cada campaña.

Otro aspecto novedoso y de cierto interés del convenio firmado por estas tres instituciones es su compromiso temporal. Con un período inicial de aplicación de cinco años, el convenio contempla sucesivas prórrogas y revisiones de los compromi- 
sos adquiridos en el desarrollo del proyecto. Esta característica permite una programación a medio y largo plazo, no sólo en lo referente a las líneas básicas del mismo que se enmarcarían en las que caracterizan la llamada Arqueología de la Muerte (Chapa et alii, 1998), sino también al asumir con cierta flexibilidad los diferentes replanteamientos que se suelen dar de una campaña a otra en cuanto a perspectiva y ámbitos. También permite planificar las posibilidades de aplicación de los cada vez más necesarios y en ocasiones determinantes protocolos analíticos.

Por último la Diputación Provincial de Toledo hace suyo el compromiso de contribuir con los medios a su alcance para asegurar y posibilitar la divulgación de los resultados de las excavaciones tanto en lo que se refiere a la publicación de los resultados de la investigación arqueológica como la integración de los mismos en el desarrollo de un proyecto paralelo que luego explicaremos en el apartado dedicado al Centro de Interpretación de la Casa Norte.

Con este esquema de trabajo la excavación del yacimiento volvía a tener sentido, aunque aún se nos planteaba el problema de la función social del yacimiento en el entorno más inmediato que, cada vez más, nos venía demandando otro tipo de actuación más permanente, accesible y explicativa, que fuera más allá de la consabida conferencia anual en el centro cultural local.

\section{LA EXCAVACIÓN}

Las campañas de excavación del yacimiento del Palomar de Pintado, con el preceptivo permiso de la Consejería de Cultura de la Junta de Comunidades de Castilla La Mancha, se llevan a cabo todos los años a partir del 18 de Septiembre durante tres semanas.

Los alumnos y alumnas participantes pertenecen a la Facultad de Humanidades de Toledo y si bien en la primera de las campañas la totalidad procedía de los cursos del $2^{\circ}$ ciclo, se ha procurado en las siguientes extender la posibilidad de acceso a ambos ciclos, lo que conlleva un cierto sistema de selección. Dado que el primer objetivo es el de su iniciación en la metodología de campo el número de participantes en cada uno de los dos turnos que se organizan por campaña no suele superar la docena. Este es un número manejable para realizar este tipo de prácticas divididos en grupos de dos,

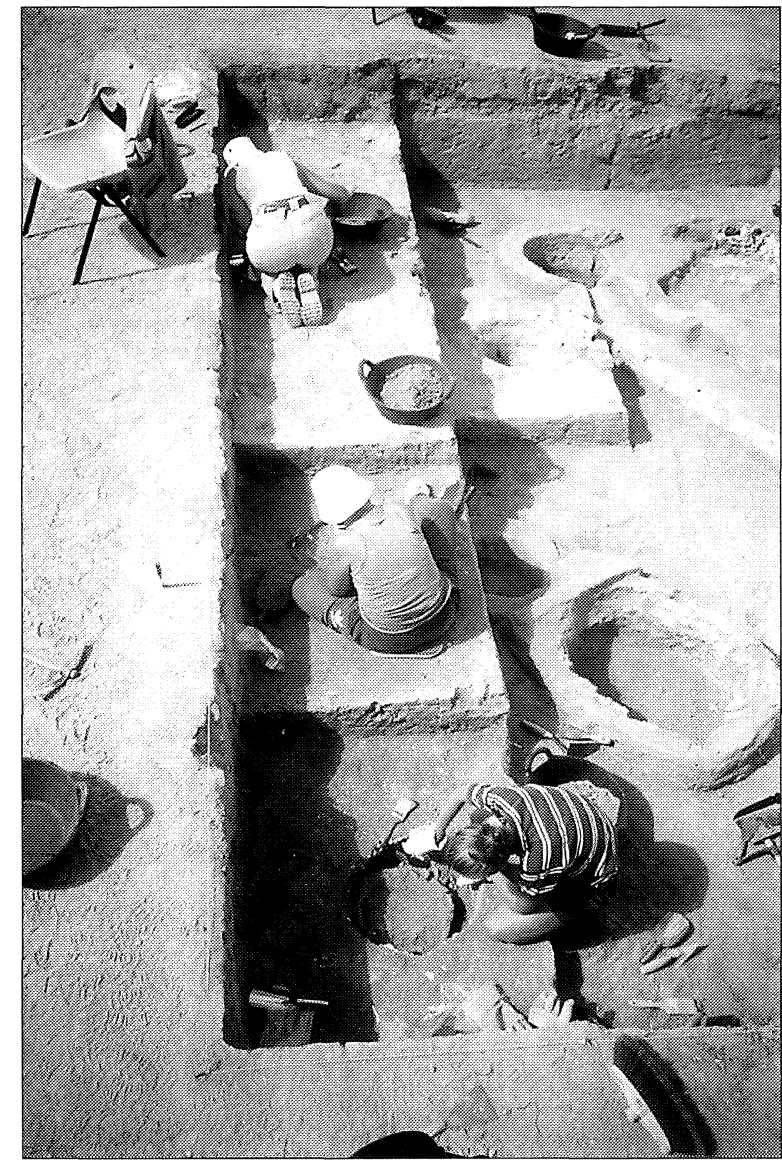

Lám. II. Tumbas en proceso de excavación. Campaña de 1999 de Palomar de Pintado (Villafranca de los Caballeros, Toledo).

tres o cuatro integrantes, según lo demanden las distintas actividades de las que reciben primero las nociones básicas teóricas, para proceder inmediatamente a su aplicación práctica: toma de profundidades, montaje de sistemas y toma de medidas para dibujo, preparación y consolidación de materiales, delimitación y excavación de unidades concretas, como fosas, hogueras, manchas, estructuras funerarias etc.

Las características del yacimiento permiten en nuestra opinión que el primero de los objetivos de este proyecto, la formación en trabajos de campo, se pueda efectuar en condiciones inmejorables. Por un lado el excelente estado de conservación de las estructuras funerarias y de los ajuares de las mismas, facilita su localización y excavación sin demasiadas complicaciones lo que permite al estudiante inexperto asumir rapidamente las tareas de excavación (Lám. II). De otro lado, la localización

T. P., 57, n. ${ }^{\circ} 2,2000$ 


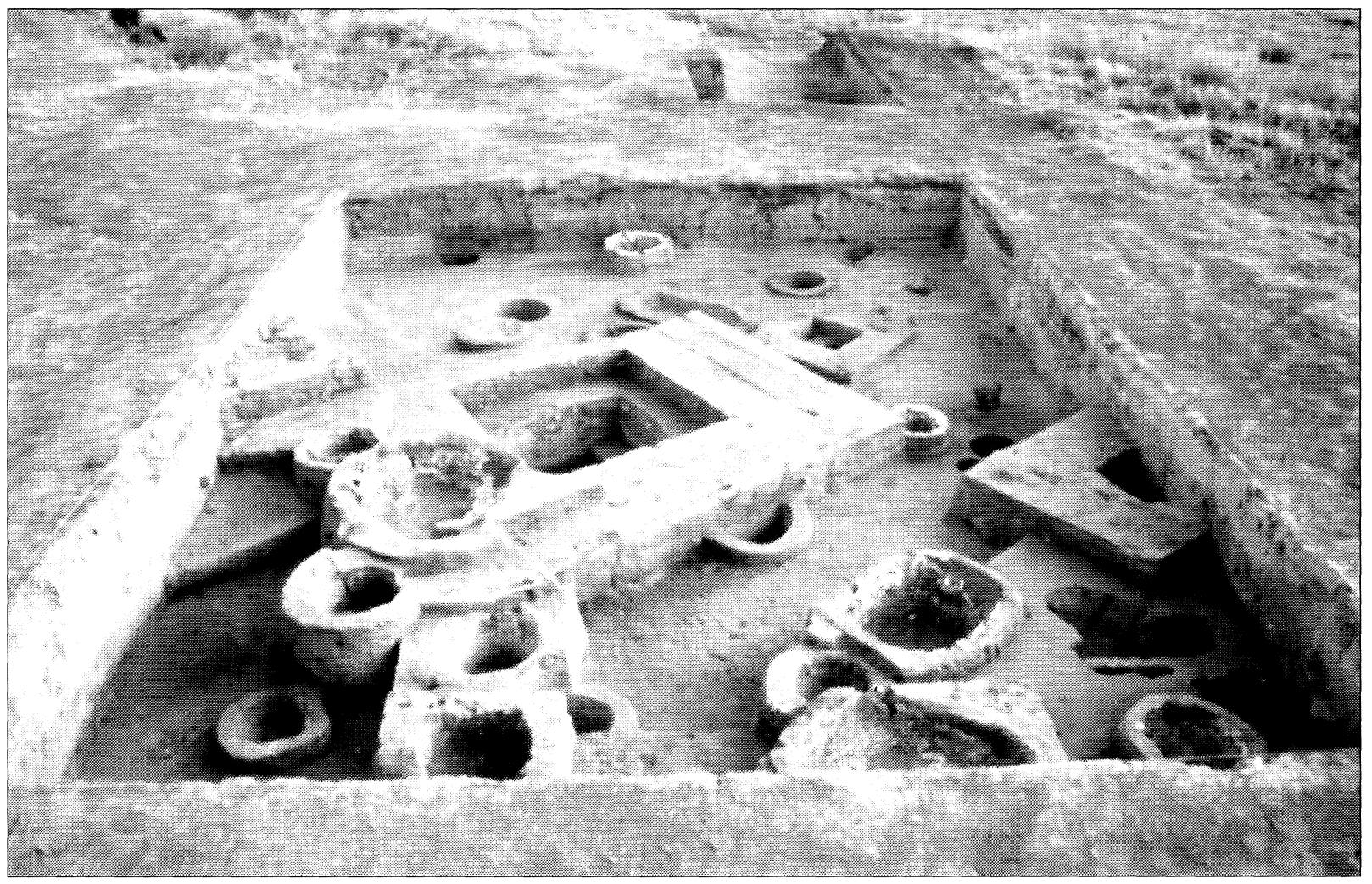

Lám. III.Vista general de la necrópolis de Palomar de Pintado (Villafranca de los Caballeros, Toledo).

y extracción de los distintos elementos del ajuar funerario, se convierte en un factor de recompensa y motivación que refuerza el proceso de aprendizaje, que hay que controlar para evitar que el interés del alumno se centre únicamente en la extracción de piezas.

Siguiendo este criterio, cada equipo que localiza una estructura funeraria pasa a ocuparse, salvo casos especiales, de todos los trabajos de excavación y documentación de la misma. Bajo el control y supervisión de los responsables de cada sector, los alumnos se ocupan de la delimitación, excavación, toma de muestras, limpieza, consolidación y extracción de los materiales. También se encargan de cumplimentar los distintos tipos de fichas de descripción e inventario, toma de medidas, dibujos de plantas, secciones y alzados, así como de los trabajos de limpieza, etiquetado, almacenaje, y transporte de todos los materiales y muestras correspondientes a la estructura funeraria bajo su responsabilidad. En los trabajos de Laboratorio que se desarrollan durante el curso académico cada grupo de excavación, cuya identificación figura en los diarios, se convierte en el último eslabón del regis- tro de la información a la hora de resolver problemas de identificación e interpretación.

El planteamiento general de la excavación pretende también, dentro de las limitaciones del yacimiento, que los alumnos perciban las diferencias y posibilidades de los dos enfoques básicos en cualquier excavación: el vertical y el horizontal y sus implicaciones de interpretación cronológica. Así en uno de los sectores del perímetro de la necrópolis delimitado por un acusado desnivel se han orientado los trabajos de excavación a la realización de un sondeo vertical con el fin de documentar el proceso de formación y desarrollo temporal de la necrópolis. Estos trabajos han permitido localizar el nivel de base de la misma caracterizado por enterramientos de incineración en urnas a mano depositadas y entibadas con piedras en hoyos de planta circular, con una cronología en torno al siglo VI a.C. con paralelos en la necrópolis de Las Madrigueras (Almagro Gorbea, 1969).

En el sector central de la necrópolis donde se vienen localizando la mayoría de las actuaciones desde el año 1986, el planteamiento de los trabajos de excavación es diferente debido por un lado al 
excelente estado de conservación en que aparecen las estructuras funerarias, incluso a pocos centímetros de la superficie, y por otro al gran agrupamiento de las mismas con múltiples adosamientos y superposiciones (Lám. III). Ante estas circunstancias el criterio que venimos siguiendo es el de no desmontar las estructuras funerarias, dotándolas de un perímetro de protección a modo de testigo que permite incluso mantenerlas en su cota , cuando los trabajos en su sector rebajan el nivel. Solo se desmontan aquellas estructuras de menor tamaño y en las que las condiciones del progreso de la excavación hacen prácticamente imposible su conservación (Carrobles, 1995: 257). Este criterio genera un "paisaje arqueológico" ficticio, que no coincide con el que se formó durante las distintas fases de utilización de la necrópolis; pero que permite mostrar a los alumnos las posibilidades del enfoque horizontal, para una lectura sincrónica de las fases del yacimiento, así como la práctica de sencillos ejercicios de cronología relativa a partir de la documentación de las distintas superposiciones.

Durante la campaña de excavaciones, junto con los trabajos de campo los alumnos participan en una serie de actividades programadas y Seminarios que se desarrollan en el Aula de la Naturaleza sobre otros aspectos del trabajo arqueológico como es el de la prospección. Este Seminario en concreto permite no sólo la iniciación en una de las actividades básicas de documentación arqueológica tanto en proyectos de investigación, como en los de la llamada arqueología de gestión impulsada por la Consejería de Cultura, sino también rentabilizar el horario de trabajo cuando las condiciones atmosféricas adversas se alían con la geografía de la zona y hacen imposible el acceso al yacimiento. Cuando ocasionalmente se plantea esta situación los alumnos realizan prácticas de prospección arqueológica superficial cuyos resultados, pasan a las fichas normalizadas proporcionadas por la Consejería de Cultura, que ha autorizado con el correspondiente permiso tal actividad.

Otra de las funciones que cumple el Aula de la Naturaleza es la de servir de sala de exposición temporal de los conjuntos y materiales obtenidos cada día, para que puedan ser visitados por los vecinos, durante las tardes y los fines de semana. Desde la primera campaña en el año 1997 nuestra actividad no pasa desapercibida en el entorno de la localidad de Villafranca, ya que nuestra presencia y el inicio de los trabajos figuran en el programa de fiestas locales. Si a esto le añadimos la coinci- dencia con las tareas de la vendimia en toda la comarca, es fácil entender el que seamos objeto de numerosas visitas, que tras la sorpresa inicial por las características de nuestro trabajo y los hallazgos realizados, demandan con inagotable curiosidad más información sobre la fecha, cultura y costumbres de la gente que utilizó la necrópolis. Un porcentaje importante de estos visitantes suelen pasarse por el Aula de la Naturaleza donde la contemplación de los distintos recipientes y elementos de los ajuares funerarios no hacen sino avivar su interés por conocer más cosas de todo lo relacionado con el yacimiento. Cabe resaltar que esta política de puertas abiertas no ha supuesto hasta el momento ningún factor de riesgo para la conservación del yacimiento, antes bien se ha convertido en una fuente de información sobre la existencia de otros yacimientos en la zona y en la certeza de que cualquier actividad incontrolada sería inmediatamente detectada y puesta en conocimiento de las autoridades.

Dentro de estas actividades de difusión y coincidiendo con el inicio del curso escolar se han organizado por las tardes para los alumnos de Primaria de la localidad una serie de visitas al yacimiento acompañados por sus profesores (Lám. IV). Estas visitas se utilizan por el profesorado como un recurso didáctico para hacer más atractivos y cercanos algunos de los temas relacionados con la arqueología y el patrimonio en el marco de la asignatura de las Ciencias Sociales.

Sin embargo dentro de las posibilidades de utilización del yacimiento en distintas actividades de difusión somos conscientes que las mismas tienen el handicap de una temporalidad breve: las tres semanas que duran las campañas de excavación. Por otro lado las características del yacimiento lo hacen de difícil acondicionamiento para ser visitado durante todo el año. Facilitar el acceso supondría una inversión económica considerabl. Las estructuras funerarias de adobe, que son tapadas al concluir los trabajos arqueológicos (Lám.V), presentan grandes dificultades técnicas para su conservación tanto al aire libre como bajo distintos tipos de cerramiento y techado, que solo se podrían abordar a partir de la delimitación de su perímetro, lo que hasta el momento no se ha conseguido. La distribución de las tumbas y sus característicos adosamientos y superposiciones las hacen de dificil comprensión para el visitante, a lo que habría que añadir la imposibilidad de exponer in situ los ajuares funerarios, salvo que se contara con un tipo de estructura que propor-

T. P., 57, n. $^{\circ} 2,2000$ 


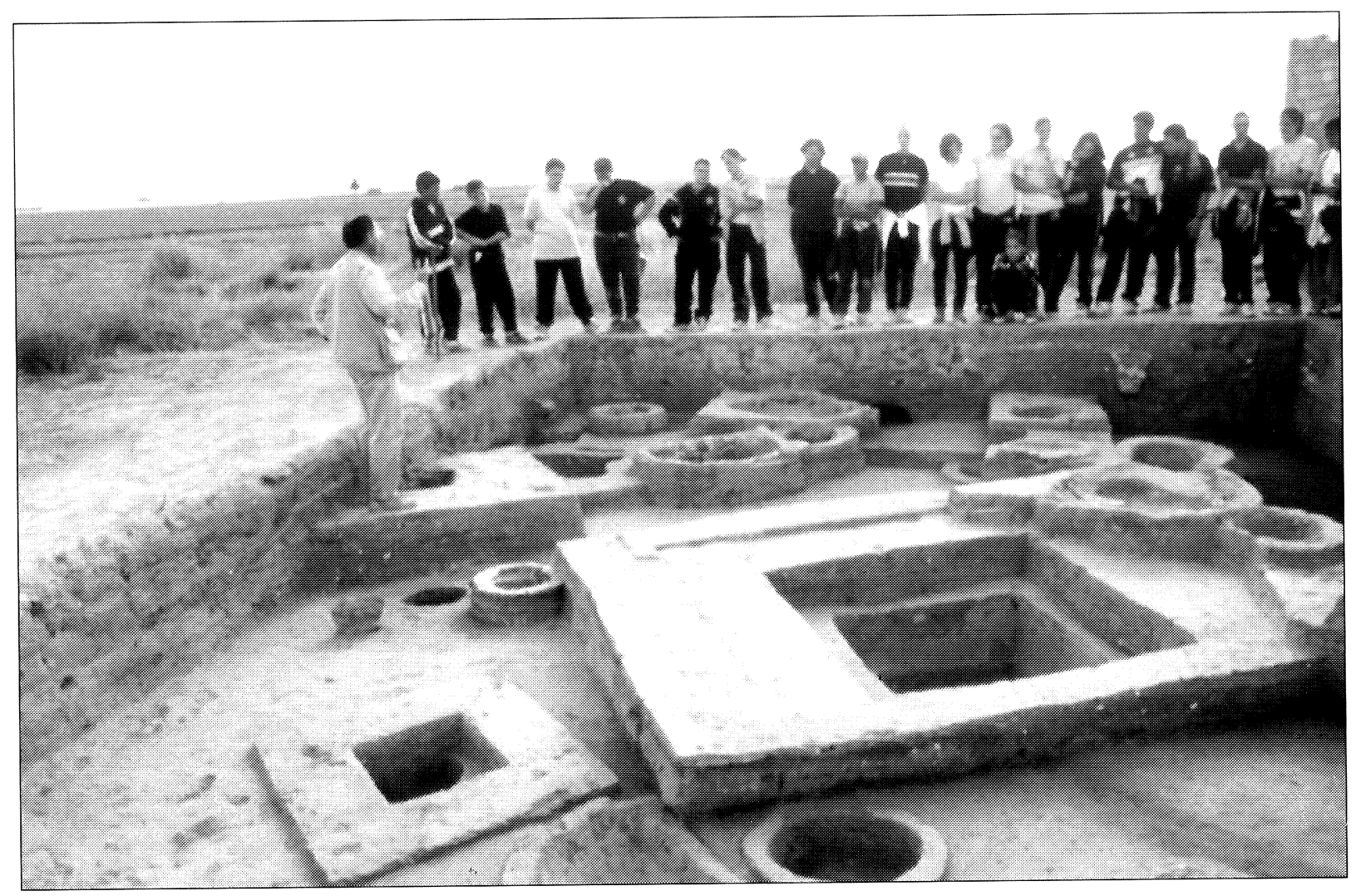

Lám. IV. El profesor Pereira “afrontando” con decisión una visita didáctica a la necrópolis de Palomar de Pintado (Villafranca de los Caballeros, Toledo).

cionara una cierta seguridad y que como se ha señalado por el momento no es viable.

La solución que permitiera conjugar la creciente demanda y expectativas de los potenciales visitantes, y las características que lo hacían difícilmente visitable fuera de la campaña de excavaciones, se perfiló al ser incluido el yacimiento del Palomar de

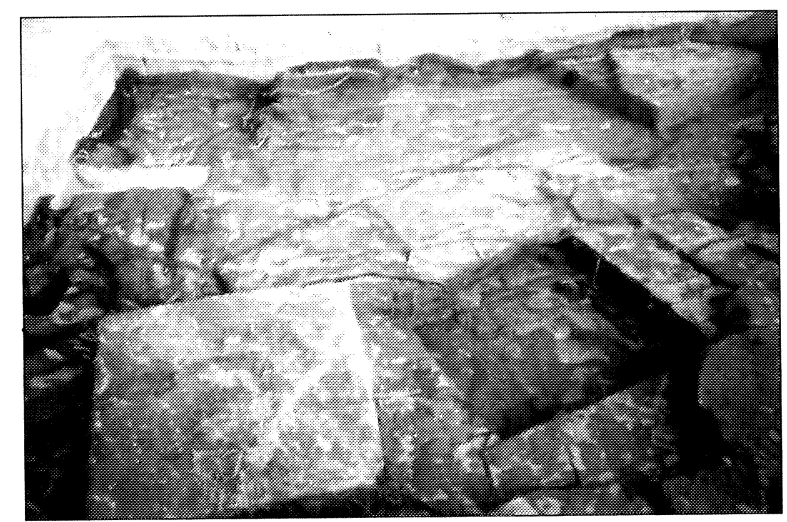

Lám.V. Sistema de protección de las estructuras funerarias de la necrópolis de Palomar de Pintado (Villafranca de los Caballeros, Toledo) antes de ser tapadas.
Pintado en el proyecto de museos locales o centro de interpretación diseñado y gestionado por el Servicio de Arqueología de la Diputación de Toledo.

\section{EL CENTRO DE INTERPRETACIÓN}

Para cumplir estas expectativas y de nuevo con la siempre necesaria colaboración del Ayuntamiento de Villafranca de los Caballeros, se iniciaron los estudios dirigidos a conocer cuál era la mejor manera de presentar el yacimiento a la sociedad. $\mathrm{La}$ naturaleza del mismo, un cúmulo de superposiciones de estructuras en adobe, alguna pequeña construcción en piedra o simples agujeros en el suelo, imposibilitaban como ya se ha reseñado la realización del acondicionamiento necesario para convertir el lugar de las excavaciones en un auténtico yacimiento visitable (Lám. VI). Este hecho, unido a la problemática conservación del resto de la necrópolis ante la posibilidad de visitas incontroladas en un lugar alejado de cualquier zona habitada, motivó el que planteáramos la ejecución de un Centro de 


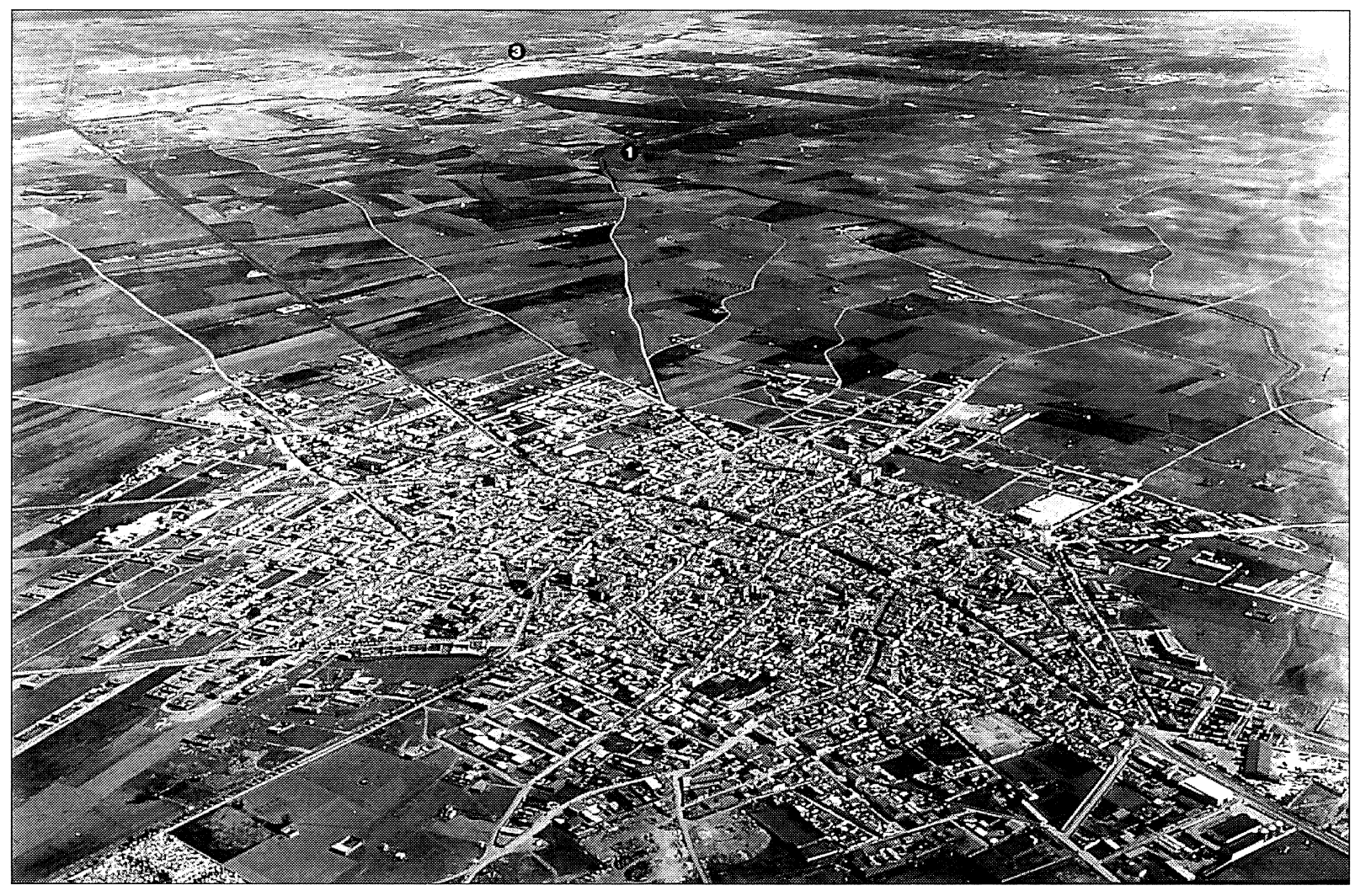

Lám.VI. Vista aérea de Villafranca de los Caballeros (Toledo). 1. Necrópolis de Palomar de Pintado. 2. Centro de Interpretación de la necrópolis de Palomar de Pintado. 3. Río Cigüela.

Interpretación del yacimiento aprovechando alguno de los inmuebles de arquitectura tradicional con los que cuenta la localidad de Villafranca de los Caballeros, dentro de su casco urbano.

De todos los inmuebles posibles el Ayuntamiento de la localidad adaptó el ubicado en la Calle del Norte que fue objeto de rehabilitación por parte de una escuela taller de titularidad municipal (Lám. VII). Tras la finalización de las obras se eligió la parte del inmueble que iba a ser destinada explicar el yacimiento de Palomar de Pintado y se dio inicio a los trabajos de montaje basado en los proyectos museológico y museográfico realizados por el Servicio de Arqueología de la Diputación Provincial de Toledo.

El primer trabajo consistió en convencer a los diferentes colectivos interesados que, en ningún momento, nos estabamos planteando la realización de un museo sino de otra figura próxima, pero con naturaleza propia, como es la del Centro de Interpretación, costeado por la Diputación y mantenido por elAyuntamiento, menos conocida por el público en general, pero mucho más efectiva para loca- lidades pequeñas en las que es imposible mantener abiertas las instalaciones que un museo requiere.

Una vez definido el producto se buscó el tipo de público en el que se quería centrar el mensaje, que se decidió fuese el del estudiante de secundaria de toda la región. Tras aclarar este particular se acometió la redacción de los diferentes proyectos que definieron un total de dos espacios. El primero destinado a plantear al visitante la cronología en la historia de la humanidad, es decir, a hacer próximos a los individuos protagonistas de la historia que les ibamos a contar en relación con los millones de años de nuestra historia, así como a situar en una barra cronológica los principales sucesos geológicos y humanos que han definido la formación de la comarca. Desde la aparición de la llanura manchega, hasta llegar a nuestros días aparecen diversos datos que permiten ubicar al visitante en el momento en el que comienza la historia que queremos contar.

Tras estos primeros montajes se llega a la sala principal en la que se desarrolla todo el mensaje que explica el yacimiento de Palomar de Pintado y, lo 


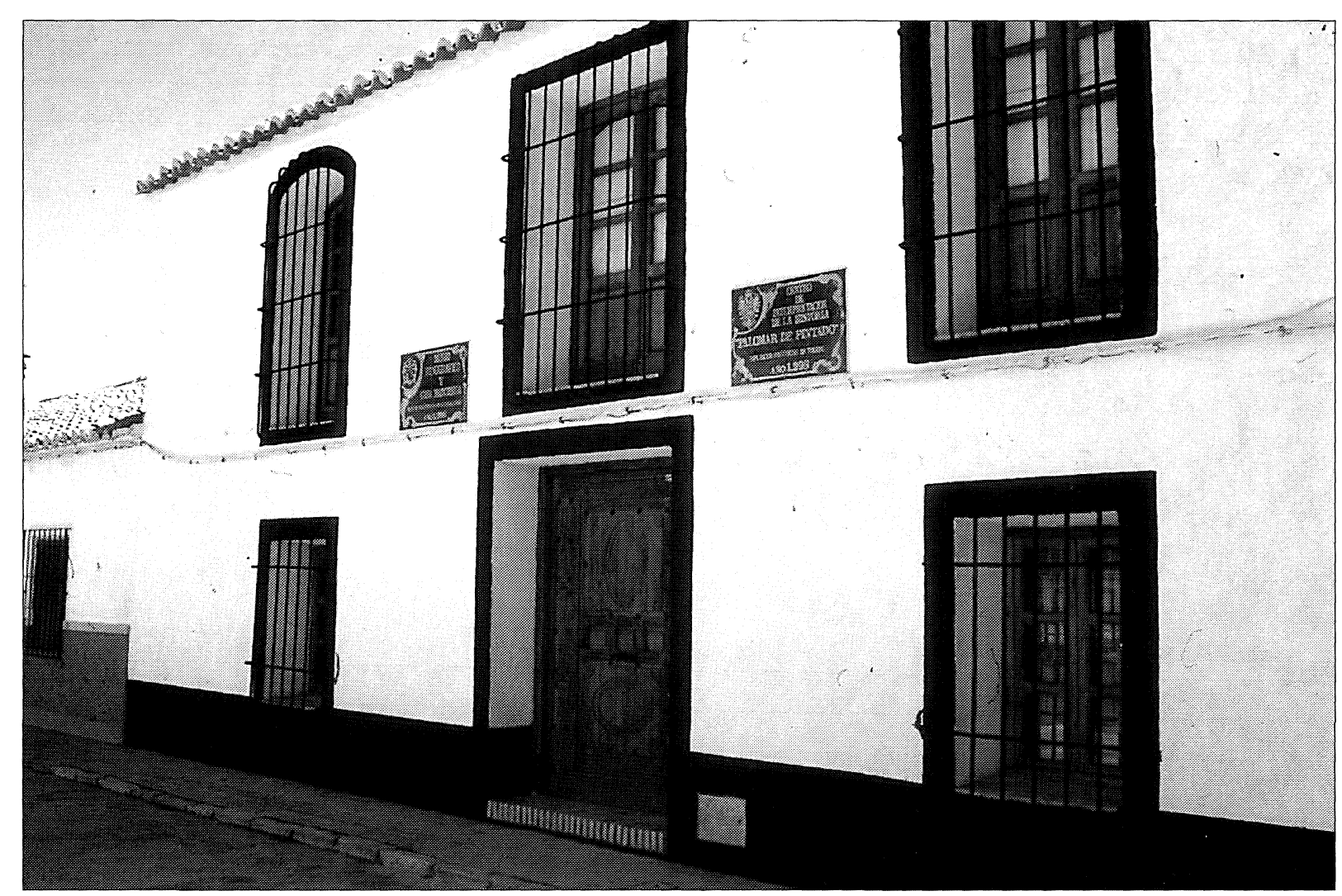

Lám. VII. Fachada de la casa típica manchega en la Calle del Norte de Villafranca de los Caballeros (Toledo) tras su acondicionamiento como Centro de Interpretación de la necrópolis de Palomar de Pintado.

que siempre fue importante para nosotros, explica cuál era el motivo de nuestra presencia en la localidad desde mediados de los años 80 , a la vez que se da a conocer los resultados de las distintas campañas realizadas. En este espacio se trata de explicar la historia del último milenio a.C. en la comarca de la Mancha, haciendo especial mención al yacimiento de Palomar de Pintado. Para conseguirlo se ideó la realización de una serie de montajes con un gran plano o mapa, acompañado de fotografías retroiluminadas y diversos textos, que posibilitaban una visita a muy diferentes niveles según pudiera ser la demanda y nivel de formación de cada grupo de visitantes. Los paneles que se incluyeron en este montaje son:

- El mundo ibérico. Origen de una cultura.

- La expansión cultural. La iberización de la Mancha.

- La necrópolis de Palomar de Pintado (Lám. IX).

- La arqueología de la muerte.
- De la aldea a la ciudad.

- Cartagineses y romanos.

- Romanos e indígenas.

- La Romanización.

- La Romanización de la Mancha.

Este conjunto de paneles que conforman el hilo conductor, se acompaña de reproducciones de los recipientes cerámicos más significativos que fueron albergadas en vitrinas instaladas aprovechando los huecos tradicionales de la vivienda. De todas las piezas expuestas merece especial interés la reproducción de la gran falcata con decoración damasquinada en plata aparecida en las últimas campañas de excavación que, ante el amplio interés que despertó en los medios de comunicación de la provincia de Toledo al ser la primera espada de hierro conocida en una zona de especial tradición espadera, ha pasado a ser, en cierta medida, la "imagen emblemática" del yacimiento.

El Centro de Interpretación abrió sus puertas en octubre del año 1998 y desde entonces se ha convertido en visita obligada para un buen número de es- 


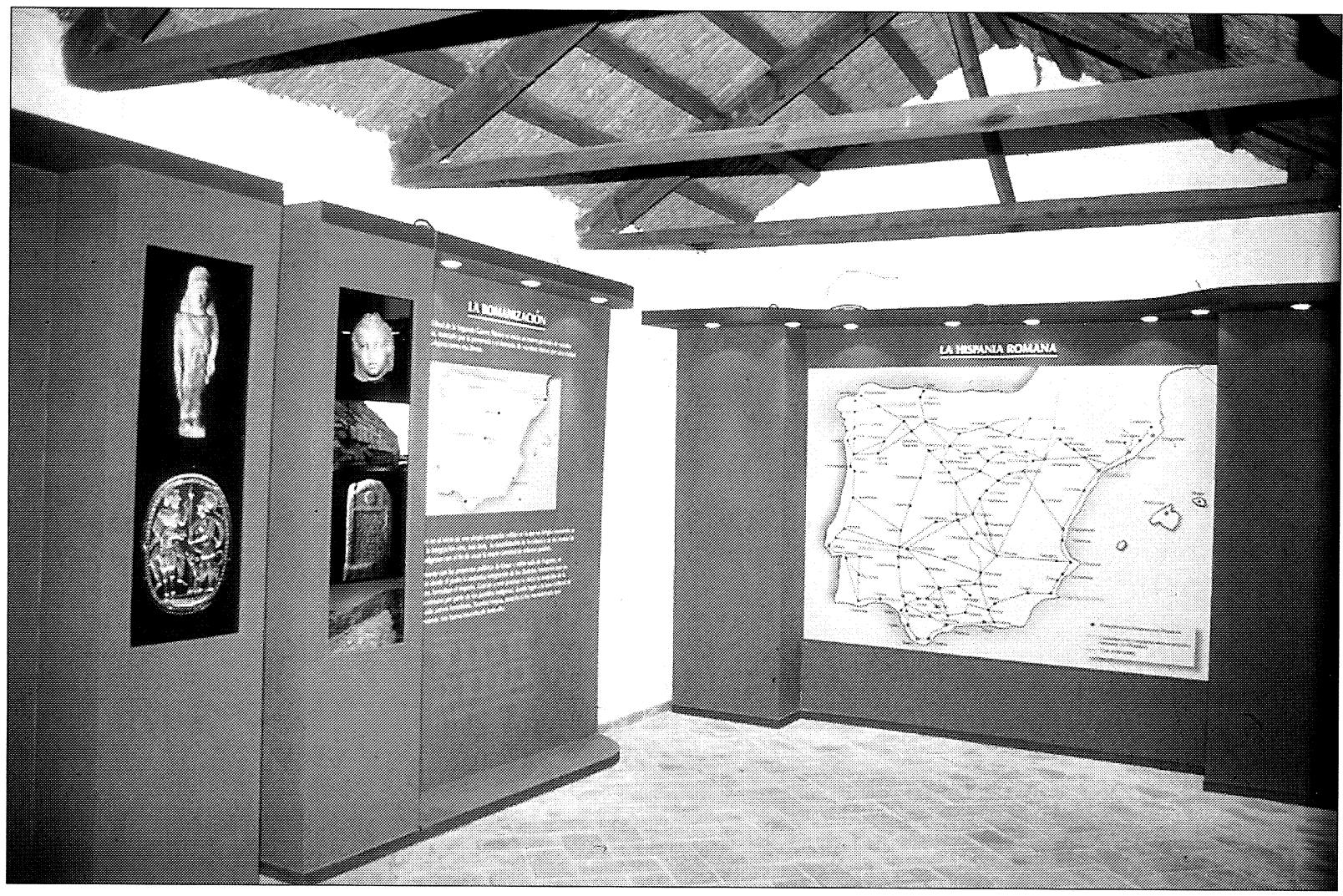

Lám.VIII. Detalle de la sala deArqueología del Centro de Interpretación de la necrópolis de Palomar de Pintado (Villafranca de los Caballeros, Toledo).

colares de toda la zona, así como en el complemento de una oferta turística incipiente que mezcla el interés por la medio lagunar manchego con la artesanía, la historia y el Patrimonio, hasta el punto de llegar a confeccionar un producto turístico que ha sido presentado en ferias tan importantes como es la Feria Internacional de Turismo Fitur 2000 en Madrid.

Este nuevo modelo basado en la colaboración institucional que posibilita la formación práctica de alumnos y que, a la vez, incide en el desarrollo local de la comunidad en la que se realiza, marca la diferencia con el viejo modelo de intervención con el que se iniciaron las actuaciones en Palomar de Pintado y suponen, a la luz de los costes y resultados, una magnífica propuesta para seguir actuando en otros yacimientos de la provincia de Toledo.

\section{CONCLUSIÓN}

Hasta la fecha el grado de cumplimiento de los objetivos del convenio por parte de las instituciones implicadas y la rentabilidad social obtenida es altamente satisfactorio. Esta situación se traduce en que el propioAyuntamiento de Villafranca de los Caballeros sea el primer interesado en plantear la intervención en nuevos yacimientos alguno de los cuales completaría la información procedente de la necrópolis. A esto hay que añadir el hecho de que todos los años se tenga que realizar un sorteo entre los alumnos toledanos para poder formar el equipo de trabajo y, sobre todo, la evidencia de que por primera vez, $\mathrm{y}$ tras tres campañas sucesivas, vuelvan a aparecer nuevos licenciados capaces de aspirar a un hueco en la práctica de la arqueología como profesión tras más de una década sin caras nuevas. Estas son las mejores cartas de presentación de un modelo de gestión en el que a nuestro juicio se integran de manera armónica los aspectos científicos y académicos con el acceso y disfrute social de un bien cultural.

La verificación de estas expectativas tan optimistas vendrá en el curso de dos o tres años, cuando tras la revisión de la prórroga se esté desarrollando una segunda fase del proyecto y la información sobre los 


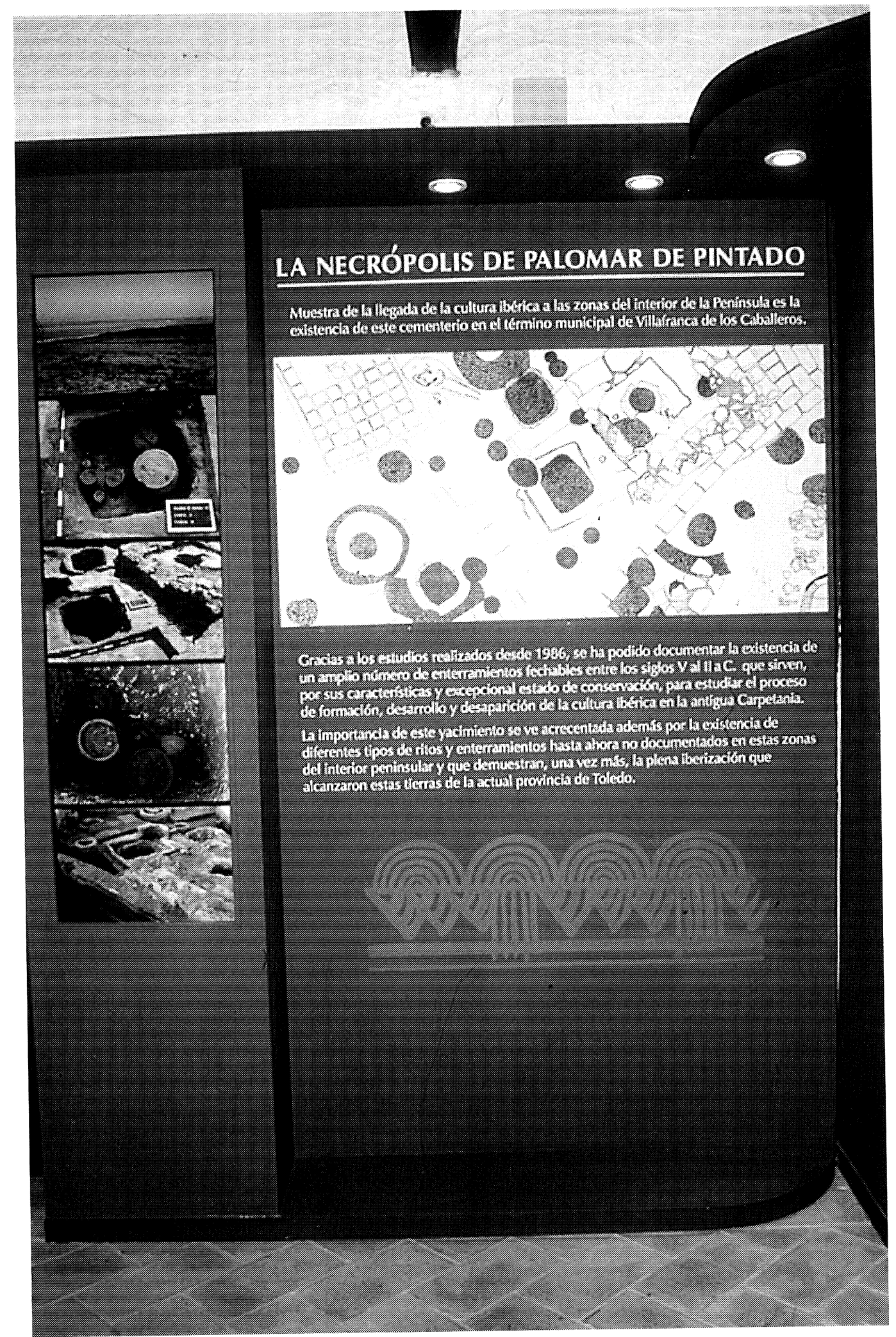

Lám. IX. Panel explicativo de la necrópolis de Palomar de Pintado (Villafranca de los Caballeros, Toledo).

T. P., 57, n. ${ }^{\circ} 2,2000$ 
resultados y características del mismo, en los distintos medios académicos, científicos y de divulgación, lleve asociada la firma de los nuevos profesionales que se formaron en el proyecto.

\section{BIBLIOGRAFÍA}

Almagro Gorbea, M. (1969): La necrópolis de "Las Madrigueras". Carrascosa del Campo (Cuenca). Biblioteca Praehistorica Hispana, X. Madrid.

Blasco, C. y Barrio, J. (1992): "Las necrópolis de la Carpetania". En J. Blánquez y V. Antona (eds.): Congreso de Arqueología Ibérica. Las necrópolis (Madrid, 1991): 279-312. Madrid.

Carrobles, J. (1995): "La necrópolis ibérica de Palomar de Pintado (Villafranca de los Caballeros, Toledo)"..En J. Blánquez (ed.): El mundo ibérico. Una nueva imagen en los albores del año 2000. Junta de Comunidades de Castilla La Mancha: 252-257. Toledo.

Carrobles, J. y Ruiz Zapatero, G. (1990): "La necrópolis de la Edad del Hierro de Palomar de Pintado (Villafranca de los Caballeros, Toledo)". Actas del Primer Congreso de Arqueología de la provincia de Toledo (Toledo, 1988): 235-258. Toledo.

Chapa Brunet, T.; Pereira Sieso, J.; Madrigal Belinchón, A. y Mayoral Herrera, V. (1998): La necrópolis ibérica de los Castellones de Céal (Hinojares, Jaén). Junta de Andalucía y Universidad de Jaén. Sevilla.

Palomero, S. y Carrobles, J. (1999): "La felicidad en los pequeños museos de sitio y el "espíritu del jaiku": algunos ejemplos en la provincia de Toledo". Museo, 4: 209-221.

Ruiz Zapatero, G. y CARrobles, J. (1986): “Una necrópolis tumular ibérica en la Mancha. Villafranca de los Caballeros (Toledo)". Revista de Arqueología, 66: 58-61. 